\title{
Importancia de la cultura de patio para la economía comunitaria
}

\author{
Xavier Ernesto Rodríguez Corea
}

\section{Resumen}

El presente artículo pretende abordar el cultivo de patio desde su componente cultural como parte de las familias y la comunidad campesina, en el marco de estrategias locales, esfuerzos colectivos y programas gubernamentales para combatir la pobreza extrema y el sistema que la produce y perpetua. La aproximación a la comunidad concreta permite conocer la particularidad pero también encontrar puentes que vinculen estas reflexiones con la realidades compartidas por buena parte de las comunidades en todo el país, especialmente aquellas en donde la herencia campesina y el vínculo con la tierra y sus frutos sigue siendo fuertes, a pesar del constante avance de la lógica urbana y de tercerización de la economía. El presente trabajo tiene su base en la etnografía como método de aproximación a la vida cotidiana y la teoría antropológica como lógica científica de compresión de la comunidad y sus estrategias de desarrollo, y se enmarca en el esfuerzo sostenido de investigación que viene desarrollado el Departamento de Antropología de la Facultad de Humanidades y Ciencias Jurídicas de la UNAN- Managua, es decir está profundamente vinculada con un esfuerzo colectivo e institucional.

Palabras claves: Antropología, Antropología económica, Antropología política, comunidad, economía, desarrollo, desarrollo humano.

\begin{abstract}
This article approaches economics from its cultural compontent, as part of the economic strategies of peasant families and communities, within the framework of local strategies, collective efforts and governmental programs to combat extreme poverty and the system that produces and perpetuates it. We reflect and link our reflections with the realities shared by a major part of the rural communities in the whole country, especially those in which the traditional peasant link to the land and its fruits continue to be strong, despite the constant advance of the urban logic and the tendency toward a service-oriented economy. This paper is based on ethnography as a method of approaching daily life, and anthropological theory as a scientific logic for comprehending the community and its development strategies. Our work is situated in the sustained research effort of the Department of Anthropology of the Faculty of Humanities and Juridical Sciences at the National Autonomous University of Nicaragua, Managua (UNAN-Managua).
\end{abstract}

Key-words: Anthropology, Economic Anthropology, Political Anthropology, Community, Economy, Development, Human development. 


\section{Introducción}

Son muchos los temas de interés que propone las ciencias sociales, concretamente la antropología propone una amplia serie de apasionantes temáticas desde el problema agrario o la identidad indígena, hasta las nuevas identidades juveniles o la interacción virtual. Son temas interesantes, necesarios y urgentes, sin embargo nos corresponde como científicos discernir aquellas temáticas que son estratégicas para el desarrollo de los pueblos y las poblaciones. Pero no sólo se trata de ¿Qué investigar? sino y también ¿Cómo hacerlo? En este sentido nos retamos a descolonizar la ciencia que es siempre un reto grande, luego de tantos siglos de colonialismo que aun hoy se reproduce, y ante esto es que nos ponemos no sólo a investigar con rigurosidad científica, sino también con perspectivas originarias, es decir desde la visión del ciudadano investigador (Jimeno, 2000, pág. 163). Que hace extraño lo propio y se apropia de la diversidad para describir, analizar y comparar la cultura de las poblaciones.

Es por eso y ante los importantes esfuerzos que hacen las familias y comunidades por generar ingresos que les permitan alimentase, vestirse, pero también apuntando hacia suplir necesidades como la recreación, la mejora de infraestructura, el acceso a los servicios, es que ponemos la mira sobre la producción campesina. Lo que se produce en el campo es importante no sólo por ser la principal herramienta de estas comunidades para salir de la pobreza, sino que también porque estamos convencidos que generan el alimento y la riqueza que en buena parte sostiene a nuestras sociedades y que producen bien-estar al resto de las poblaciones y que lamentablemente pasa desapercibida de los análisis de buena parte de los economistas y científicos sociales.

En los últimos años el Gobierno de Unidad y Reconciliación Nacional ha orientado importantes esfuerzos al combate de la pobreza y la pobreza extrema especialmente en el sector rural. Según la Encuesta de Medición de Nivel de Vida del Instituto Nacional de Infor mación de Desarrollo (INIDE) para el período 2009 a 2014 en Nicaragua hubo una disminución del 13 por ciento en la pobreza nacional, mostrando una disminución del 42.5 al 29.6 por ciento. Mientras que la pobreza extrema presentó una disminución del 6 por ciento, donde bajó de 14.6 a 8.3 por ciento" (INIDE, 2014). Sin embargo aún las comunidades rurales presentan serios retos en la consecución efectiva de sus derechos humanos. Nuestro deber como intelectuales es aportar desde la perspectiva científica a la compresión profunda de la comunidad como escenario real de los hechos, es aproximarnos a la vivencia cotidiana, con un ojo en la práctica y otro en la teoría, hacer la lectura de las propuestas y alternativas que nos ayuden a seguir caminando, en la búsqueda de la siempre retadora relación teoría-práctica.

Para conocer la experiencia de producción de una comunidad campesina, nos aproximamos a El Arenal, una pequeña comunidad de Masatepe, departamento de Masaya, donde al igual que en otros contextos rurales, existe una pervivencia de los elementos indígenas y de la cultura campesina. Una identidad que sobrevive a pesar de la cercanía de los centros económicos urbanos como Masatepe, Masaya y Managua, a pesar de las migraciones, las remesas y otros fenómenos de cambio cultural que apuntan hacia una creciente urbanización del espacio físico pero también de las relaciones sociales.

En nuestras comunidades campesinas se libran luchas, por el agua, la salud, el medio ambiente, pero también por la sobrevivencia diaria y en este marco hay que destacar la importancia de lo que se produce en el patio para la economía de la familia. Un hecho que es totalmente coincidente con la propuesta del desarrollo humano como paradigma emergente en América Latina en sus connotaciones prácticas, pero también éticos políticos, es decir el desarrollo que tiene en el centro al ser humano y que busca algo más que crecimiento económico y que de hecho lo pone en segundo plano, un desarrollo vinculado con el disfrute y la autorrealización personal y colectiva.

Nos proponemos en primer lugar aproximarnos a una comunidad concreta y desde allí compartir algunos hallazgos empíricos que nos permiten comprender algunas implicaciones del cultivo de patio en la economía y la identidad de la comunidad, estableciendo respectivas vinculaciones con diversos aspectos de la cultura local.

El Arenal, en Masatepe, es un municipio del departamento de Masaya, caracterizado en lo productivo por una amplia variedad de rubros agrícolas, en lo identitario por su estructura social con características campesinas, vinculadas por fuertes lazos parentales y redes de reciprocidad inter-parentales, que generan fenómenos culturales muy particulares en lo educativo, religioso y organizativo, que hacen de la vida comunitaria 
tan atractiva y retadora en términos antropológicos. Así por ejemplo, los fenómenos de intercambio de alimentos y trabajos asociados a las fiestas patronales, los pequeños negocios y emprendimientos comunitarios, las organizaciones que atienden necesidades básicas como adquisición de medicamente a costos solidarios o cooperativas de ahorro y crédito, entre otras muchas expresiones no sólo son interesantes, sino vitales para la sobrevivencia de las personas.

\section{Materiales y métodos}

Una advertencia importante sobre este artículo es que debe ser visto como una fotografía tomada desde un ángulo particular, desde este impreciso instrumento llamado investigación etnográfica, que aunque impreciso sirve para que otros y otras se vean reflejados o contrastados y para que ellos y ellas mismas continúen su proceso de reflexión sobre su comunidad.

Al igual que la fotografía, la investigación social queda superada por la realidad y se desactualiza en el segundo siguiente, sin embargo sirve para motivar la reflexión científica y sobre todo la valoración emotiva de las luchas y procesos, tan importantes en el espíritu de un pueblo que durante los últimos años ha visto como da pasos certeros en la búsqueda de mejores condiciones de vida.

En el marco del método etnográfico, las técnicas son las herramientas concretas, para entender el mundo investigado y la forma general desde donde el investigador lo aborda. Así un antropólogo utiliza la técnica en función de las características particulares de la situación estudiada, aunque existen lógicas y directrices sobre la realización de la entrevista y la observación desde la antropología y desde otras ciencias que han adoptado como suyas estas técnicas, rara vez estas directrices pueden ser aplicadas al pie de la letra. Cada investigador afinará el instrumento en función de su objetivo y su subjetividad.

"En completo contraste con la entrevista estructurada, las entrevistas cualitativas son flexibles y dinámicas, las entrevista cualitativas han sido descritas como no directivas, no estructuradas, no estandarizadas y abiertas. Utilizamos la expresión "entrevistas en profundidad" para referirnos a este método de investigación cualitativo, por entrevista cualitativas entendemos reiterados encuentro cara a cara entre el investigador y los informantes, encuentros estos dirigidos hacia la compresión de las perspectivas que tienen los informantes respecto a sus vidas experiencias o situaciones tal como las expresan con sus propias palabras" (Taylor \& Bogdan, 1998, pág. 100)

De forma que la entrevista no puede, entenderse como herramienta estática sino por el contrario constantemente acomodada al momento incluyendo las diversas etapas de la investigación:

"En la primera etapa del trabajo de campo, la entrevista antropológica sirve para descubrir las preguntas, esto es, para construir los marcos de referencia de los actores a partir de la verbalización asociada libremente. Desde estos marcos se extraerán, en un segundo momento y tras una categorización diferida, las preguntas y temas significativos para la focalización y profundización” (Guber, 2005, pág. 143)

En lo referido a la observación como técnica antropológica es posible distinguir diversas aportaciones que han ido surgiendo en el desarrollo histórico de la ciencia antropológica:

"Tradicionalmente su objetivo ha sido detectar los contextos y situaciones en los cuales se expresan y generan los universos culturales y sociales, en su compleja articulación y variabilidad. La aplicación de esta técnica o, mejor dicho, conceptualizar esta serie de actividades como una técnica para obtener información, se basa en el supuesto de que la presencia - esto es, la percepción y la experiencia directas - ante los hechos de la vida cotidiana de la población en estudio - con sus niveles de explicitación - garantiza, por una parte, la confiabilidad de los datos recogidos y, por la otra, el aprendizaje de los sentidos que subyacen tras las actividades de dicha población" (Guber, 2005, pág. 115)

Desde estas dos herramientas con sus posibilidades es posible adentrarse en la cultura local para desentrañar los significados culturales y comprender las dinámicas y lógicas internas de esta comunidad, teniendo presente que en la práctica ambas herramientas deben ser naturalizadas, es decir partir de las situaciones concretas en las que se utilizan, por ejemplo la entrevista etnográfica algunas veces se realiza en el recorrido de un bus o mientras el entrevistado realiza labores propias y la observación va y viene entre directa y participante durante una festividad religiosa.

Asumimos la investigación social cualitativa desde la perspectiva etnográfica que permitirá conocer las prácticas y desentrañar los significados desde el punto 
de vista de los actores locales, en este sentido las técnicas de la entrevista y la observación se conjugan perfectamente para lograr estos objetivos, incorporamos también técnicas participativas desde la construcción colectiva del conocimiento, que aunque no son una novedad en la investigación social, sí lo es en algunos términos para la investigación antropológica.

La etnografía permite conocer desde la perspectiva de la propia población las características de su producción familiar ¿Qué? ¿Cómo? ¿Para qué se produce?, cotejando lo dicho con lo observable y entre las distintas percepciones: amas de casa, líderes políticos, religiosos, jóvenes, adultos, que desde sus puntos de vista ayudan a entender los fenómenos.

\section{Resultados}

Masatepe es un municipio del departamento de Masaya, ubicado a 53 kilómetros de la capital de la República de Nicaragua. Según la ficha municipal del (INIFOM) Instituto Nicaragüense de Fomento Municipal "El nombre del municipio de MASATEPE es originario del idioma Chorotega o más bien del idioma Nahuatlaca o antiguo mexicano. Está formado por las voces Mazalt, que significa venado o Tepec y Tepelt, que significa cerro o lugar; es decir que MASATEPE significa lugar donde hay venados" (INIFOM, 2014)

Masatepe se encuentra en el marco geográfico y cultural de una serie de pueblos conocidos como "Pueblos blancos" caracterizados por ser poblaciones con antecedentes indígenas, con una amplia producción artesanal y artística, en el marco de un amplio y rico patrimonio natural con fuentes hídricas, suelos fértiles y abundante flora y fauna, también cuentan con muchas expresiones culturales como: fiestas patronales, danzas, música, gastronomía y prácticas cotidianas, que hacen de estos pueblos contextos ideales para el turismo nacional e internacional.

Entre los llamados "Pueblos blancos" podemos identificar, municipios y comarcas que aunque vecinos de la ciudad de Managua, guardan grandes distancias ecológicas y culturales con la ciudad capital y los hacen distintivos frente al resto de las regiones geográficas y culturales. Diría, Diriomo municipios de Granada, San Juan de Oriente, Catarina, Niquinomo, Nindiri, Nandasmo y Masatepe, La Concepción correspondiente al departamento de Masaya y Ticuante del departamento de Managua, todos asentados en la Meseta de los Pueblos, comprendida en la llanura Adriana entre las coordenadas $11^{\circ} 55^{\prime}$ de latitud Norte y $86^{\circ} 08^{\prime}$ de longitud Oeste.
El contexto donde se desarrolló esta investigación está ubicado a siete kilómetros al Norte de la ciudad de Masatepe, hasta la comunidad se llega desde el propio Masatepe, el medio más usual para llegar hasta El Arenal es la moto-taxi. A pocas cuadras del parque central y la iglesia catedral frente al parque Veracruz, se reúnen las personas que viajan hacia El Arenal. Este lugar es un punto de encuentro y socialización, hasta este lugar llegan las personas a buscar estos vehículos que especialmente hacen este recorrido, pues aunque en Masatepe es posible encontrar gran cantidad de moto-taxis llevando y trayendo pasajeros, sólo algunas se aventuran a realizar este recorrido en gran parte por el deteriorado estado del camino.

Una de las principales características de esta y otra comunidades campesinas de la zona es la creciente urbanización de la vida cotidiana, esto se puede evidenciar en los cambios en el espacio físico, como la estructura de las viviendas, el acceso a ciertos servicios, especialmente el de las comunicaciones y los usos cotidianos de la población, pero también pueden ser evidenciados desde la misma población en los procesos de identificación y auto-identificación, así por ejemplo el uso de la palabra comunidad o comarcas para referirse a estos lugares está cayendo en desuso y en su lugar se usa barrio y se asocia esto con el "avance" el "progreso" de un lugar.

Estos cambios en la cultural local contribuyen a generar un proceso de des-campesinización que resulta aún más evidente en el plano económico y material con la tercerización de la economía campesina. Las y los pobladores asumen como natural y deseado "dejar de ser campesinos" y esto implica incurrir cada vez más en actividades que generen ingreso a la familia y que estén vinculadas con el mundo urbano.

"Cuando los y las jóvenes salen de la comunidad ven un mundo diferente, y al ver ese mundo diferente, hace que ya no te guste El Arenal, ya no se quieren empolvar, hace que ya querrás tener una casa no con piso sino con cerámica, un televisor no blanco y negro sino un plasma de no sé cuentas pulgadas, incluso ahora ya no es ver programas nacionales, ahora es cable, tener una computadora, sino cable, internet..." (Sevilla Alemán, 2014)

De forma que los cambios en lo que se produce y cómo se produce están directamente relacionados no sólo con los aspectos materiales o económicos, sino también con los aspectos identitarios culturales que son pocas veces abordados e inclusos desestimados 
en muchos casos por los tomadores de decisión. Este aspecto es desestimado en muchas investigaciones e intervenciones por considerarlo poco importante o subjetivo, lo cual plantea un reto que este artículo pretende abordar, el reto de valorizar el componente cultural en los procesos productivos.

Sin embargo es también importante apuntar experiencias exitosas en el plano local y estatal, donde se asume la importancia de las prácticas culturales campesinas y los saberes campesinos en la búsqueda de mejores condiciones de vida, experiencias que desde su sencillez y su magnitud demuestran la urgencia del rescate de los elementos identitarios campesinos y que al usar este capital correctamente podemos logar cambios importantes en la comunidad. En lo local podemos mencionar la iniciativa de un grupo de jóvenes organizados en la comunidad miembros del Grupo Solidaridad Arenal (GRUDESA) quienes es tán trabajando en la creación de un vivero que provee plantas frutales para la comunidad, pero que al mismo tiempo intenta rescatar en los jóvenes, que salen de la comunidad por trabajo o estudio, la vinculación con la tierra como un elemento identitario.

Sin embargo hay que decir que la comunidad aún conserva muchas prácticas propias de la lógica campesina originaria, una de ellas es el uso de la vivienda con fines productivos, pues sin importan si la familia tiene $o$ no las tierras de siembra y producción agrícola, todas las familias de la comunidad conservan en los jardines y patios un espacio privilegiado para la producción de alimentos, plantas para el ornamento y plantas medicinales.

\section{Dinámicas productivas de la población en torno al patio}

Como se apuntaba anteriormente la comarca El Arenal y otras de la zona comparten elementos culturales de origen indígena y campesino, la herencia indígena fue sepultada, pero no desaparecida, por las prácticas coloniales anteriores y contemporáneas. La herencia campesina está viviendo un proceso similar en nuestros tiempos, estas comunidades que nacen vinculadas a la tierra ven amenazado hoy su estilo de vida y poco a poco sustituido por la "modernidad urbana" que atenta en aspecto como la alimentación, las relaciones de reciprocidad comunitaria y familiar.

Recorriendo los caminos de El Arenal, es posible apreciar un cambios en la construcción de viviendas, prin- cipalmente perceptible en la ampliación de las viviendas y también el uso de materiales como bloque, zinc, láminas de diversas materias para la creación de nuevos espacios habitacionales, esto según la población asociado al crecimiento de la familias, pues cuando las unidades domésticas van creciendo, especialmente con asentamiento de nuevas unidades domésticas por el matrimonio y el nacimiento de los hijos, las tierras poseídas se van distribuyendo de forma que en cada generación se reduce paulatinamente es espacio que acompaña la vivienda.

Esta práctica de uso y división de la tierra, hace que el espacio para la siembra sea cada vez más estrecha, algunas familias poseen tierras según los entrevistados, es común que las familias de la zona posean entre 1 y 5 manzanas para la siembra, mientras otras alquilan o venden su fuerza de trabajo, sin embargo y a pesar de las posibles diferencias una constante es el uso del espacio conocido como patio, es decir el espacio circundante a la vivienda.

El patio sigue siendo el espacio para la siembra de las plantas de primera necesidad de todas las familias, las plantas de uso común y cultivo regular cuales se pueden dividir en tres grandes categorías: medicinales, ornamentales y alimenticias.

Entre las plantas y árboles medicinales se encuentran algunas como salvia, zacate de limón y eucalipto, otras plantas medicinales también tienen una función en la cocina como la hierbabuena y el culantro, otras en cambio sus frutos son usados como alimento y sus hojas con fines medicinales como el mango, la guayaba, la naranja agria. Los cajones, maseteros o jardines de hierba buena y chiltoma ubicados cerca de la cocina denotan su importancia de estas en la preparación de los alimentos diarios, por otro lado los patios de las viviendas completan su belleza con los variados colores de flores y plantas ornamentales como la flor de avispa, ave de paraíso y rosas entras muchas que regularmente se asocian con costumbres religiosas y seculares en el plano público y privado- familiar.

Estas y otras plantas presenten en los patios por grandes o pequeños que sean, representan una herramienta importante en la economía doméstica de la población, durante las entrevistas los pobladores aducían a los costos de estos productos medicinales y comestibles en las ventas de la comunidad o en los mercados locales, ¿Cuánto cuesta una naranja agria en el mercado? "Una ramita chiquita de hierba buena le quitan 
hasta cinco pesos" o como lo refiere Nicolasa Pérez, una mujer que tiene una historia de participando con diversos proyectos y organizaciones y que además se asume como productora y comerciante:

"Aquí en los patios sembramos chayote...lo que también se cultiva es la ruda, se lleva al mercado... cualquier plantita que se siembre y allí está el dinerito guardado, no en cantidades pero de eso nosotros sobrevívenos, igual toda la gente de la comunidad de una u otra forma de eso sobrevive..." (Perez Gutierrez, 2014)

De forma que podemos afirmar que la población valora el uso medicinal o alimenticio de estas plantas, pero también existe claridad en la importancia económica de estas plantas, ya sea en el ahorro de recursos económicos o en su obtención por medio de la venta. En algunas viviendas se comercian los productos del patio con las pulperías de Masatepe, en otros casos y en mayores cantidades se comercia en los mercados de las localidades cercanas.

Los árboles de mango, jocotes, naranjas, guanábanas, mamones, zapote, entre otros, representan una importante fuente de alimento y nutrientes para las familias, especialmente para las niñas y niños que asocian estas frutas con la recreación y esparcimiento, estas frutas representan el dulce de las mañanas y tardes.

Menospreciar la importancia de estos árboles para la seguridad alimentaria de las familia y especial de la niñez sería un error grave, pues como sucede en muchos espacios urbanos los árboles serian visto como obstáculo para nuevas construcciones, en cambio en estos contextos rurales los árboles frútales representan una importante fuente alimenticia para la población.

La producción de alimentos, entre ellos frutas y verduras resulta fundamental para la nutrición de las familias campesinas, principalmente porque en muchos casos la producción del patio está menos condicionada a factores externos a la comunidad, así pues, no se depende de la banca para préstamos, de la fluctuación de los precios del mercado internacional o incluso de factores climáticos, esta producción a muy pequeña escala podría asumirse como la base de la alimentación familiar, incluso antes de la producción de granos básicos.

Algunas familias se dedican al cultivo de frutas y verduras para la comercialización, es común desde horas tempranas ver salir de la comunidad los canastos de frutas, especialmente cítricos que se dirigen a los mercados de: Masatepe, Masaya o Managua. Muchos de estos productos provienen de los patios de las familias y se comercian según la temporada por varios canales: en algunos casos son llevado hasta los mercados directamente por alguien de la familia, en otros casos son vendidos a alguien de la comunidad que los lleva al mercado, en ambos casos la circulación de estos productos dentro de la comunidad no sólo se rige por la lógica comercial de oferta y demanda sino y también por las relaciones de reciprocidad intra e inter familiares.

Otra importante fuente de ingresos económicos y nutricional familiar lo representa la crianza de animales, principalmente cerdos y gallinas, en ambos casos forman parte importante de estrategias económicas y alimenticias. En el caso de cerdo es visto como una forma de ahorro, un cerdo pequeño puede costar C $\$ 200$ (doscientos córdobas) en dependencia del tamaño y la raza, sin embargo es un inversión accesible para la mayoría de las familias que luego de un arduo trabajo de crianza y cuidado, que en algunos casos puede incurrir en gastos de medicamentos, puede ser vendido después de un par de meses en C $\$ 1,500$ (mil quinientos córdobas) , si la familia decide destazar al animal y vender sus productos la cantidad obtenida puede ser aún mayor.

Muchas familias crían cerdos para con su venta o su destace obtener importantes recursos para actividades festivas, en otras ocasiones por encima de los ingresos económicos prevalece la necesidad de compartir el producto con la comunidad por una celebración familiar, por promesas religiosas o fiestas seculares.

La comercialización de la carne de cerdo o sus productos derivados representan para muchas familias una actividad económica permanente, hasta la comunidad llegan personas en búsqueda de estos productos, chicharrón, frito de cerdo o moronga son los principales productos que se comercian en la zona y que se constituyen en plato habitual para fiestas comunitarias o familiares, de forma que muchas familias estarían interesadas en el consumo de estos productos en determinados ocasiones festivas.

En el caso de la crianza de gallinas, esta actividad es muy habitual en los hogares que con un patio amplio les posibilita la crianza de varios de estos animales. La gallina está asociada con el consumo de los huevos y del mismo animal en determinadas ocasiones, 
es común que a la llegada de una visita se dé la orden de matar una gallina, esta es una decisión que implica reconocimiento social a la ocasión pero también el sacrificio de una fuente diaria de alimentos, este cálculo entre los socialmente esperado y lo racional en términos económicos representa todo un tema de discusión y debate académico, sin embargo en la comunidad resulta hasta imperceptible en el medio de la vida cotidiana, el cálculo cultural y racional de ¿Cuántas gallinas tenemos? ¿Cuántos huevos pones? ¿Qué animal debe ser sacrificado? ¿Cuántos huevos se dejarán de producir?

Una de las actividades productivas asignadas a los niños y niñas es el cuido de las gallinas, muchas de estas le son entregadas en propiedad a los infantes para que estos "aprendan a cuidar los animalitos" este es una importante método de socialización primaria que implica el aspecto económico y de identidad que lo vincula con su origen pero también con su aporte o su papal en la economía familiar.

Además del impacto positivo que tiene la cultura de patio en las economías domésticas y de la comunidad, es importante visualizar el trabajo no salariado que le invierten principalmente las mujeres, pero donde también participan los niños, niñas y los hombres adultos y que permite que este espacio se convierta en privilegiado para la economía de la familia y la comunidad. La seguridad alimentaria y la trasmisión cultural de la herencia campesina y ancestral se enfrentan a una lógica urbana, no sólo en lo referido a las viviendas, sino ante todo a los patrones de comportamiento y los ideales de "vivir mejor" "desarrollo" y "progreso" que calan profundo en las vidas cotidianas de la población. Como se ha advertido los pesos y contrapesos que se desarrollan entre elementos materiales y espirituales son complejos y diversos, así los mismos pobladores advierten el problema de la tierra no sólo como en su aspecto identitario, sino también con fuente de conflictos social:

"En la comunidad lo que veo yo un poco más problemático, cuando los padre están heredando a los hijos, porque el crecimiento poblacional es grande, los padres es poco lo que tienen y se va distribuyendo, pero a veces si no tiene posibilidad de pagar entre la familia, el que puede le quita su herencia, por que como no tiene recursos, o si no están propiedades están allí sin darle una salida, somos gente que simpatizamos al Partido, pero cuando vamos a hacer la diligencias eso lleva ingresos tremendos con los abogados y con las instituciones y la pasibilidad que hay en la atención, que la renta que el este, que el otro, se te pasa el tiempo y se te vuelve a multiplicar, así está la situación, eso de la propiedad lo veo bastante complicado" (López Mercado, 2014)

Junto al problema del crecimiento población, están apareciendo los conflictos por la propiedad entre las familias y entre pobladores, esto debido que a que contrario a tiempos anteriores, la tierra resulta un bien escaso, debido a la división de lo que antes era un terreno amplio en parcela cada generación más pequeña. En estos momentos estas situaciones destacan en la comunidad por ser cada vez más común, este fenómenos del crecimiento urbano y escasez de la tierra motiva la migración y la utilización de otras estrategias económicas como el comercio, la prestación de servicios o la venta de la manos de obra en los centros urbanos, especialmente por parte de los más jóvenes.

\section{Discusión de resultados}

La comunidad campesina es espacio privilegiado para el estudio del fenómeno de cambio-continuidad presentado en términos productivos, donde las personas se organizan y desarrollan acciones y estrategias para mejorar sus condiciones de vida y las formas en que las unidades familiares obtienen lo necesario para la sobrevivencia por medio de la producción agrícola y en ambos casos la influencia de los procesos de "modernización" impuestos-propuestos por el sistema capitalista, sin embargo se carece de una caracterización de los procesos económicos que se desarrollan en el territorio, en base a lo anterior:

La economía se define, entonces, como una serie de relaciones sociales presentes tanto como un ámbito de actividades específico el de la producción, distribución y consumo de bienes materiales como un "aspecto específico de todas las actividades humanas que no pertenecen, en sí mismas, a dicho ámbito, pero cuyo funcionamiento implica el intercambio y la utilización de bienes materiales" (Narotzky, 2004, pág. 5)

En el caso de la producción de frutas, verduras y granos básicos del contexto de estudio, la categoría economía resulta pertinente y acertada, sin embargo podemos retomar para la comprensión de la economía local una propuesta que más allá de un concepto nos aporta una relación teórica- metodológica en la búsqueda de los significados de la economía local y que establece relación con la cultura de patio en cuanto: "[...] la comprensión antropológica de los diferentes 
modelos económicos locales o nativos no debería estar guiada por la inclusión de estos en de-terminadas categorías occidentales de conocimiento sino por la búsqueda de sentido dentro de la configuración cultural concreta: desde su posición un modelo es una construcción cultural, tanto de creencias como de prácticas, y no una reproducción antropológica de los hechos basada en ciertas determinaciones económicas a priori, la comprensión antropológica de un modelo económico se debe basar en las capacidades, el conocimiento social, la experiencia o las diferentes conexiones que se dan entre los individuos implicados en él y en cómo se define, se crea y se distribuye el valor -algo culturalmente definido" (Pinto, 2007, pág. 65)

Esta propuesta de contextualización de la economía, es en realidad una propuesta profundamente teórica por que orienta la reconstrucción de los modelos existente en el marco de los hallazgos etnográficos locales. Es decir la producción de economía campesina y concretamente la cultura de patio responde a un modelo particular el cual debe ser explicado desde sus implicaciones culturales incluyendo la racionalidad del uso de los recursos y las prácticas económicas cotidianas como el intercambio, las actividades domésticas y su valor en la economía

Por otro lado podríamos también aproximarnos a una categorización amplia que nos permita una categorización más general. En este sentido, Gudeman se refiere a cuatro dominios de la economía comunitaria:

"Nos encontramos en primer lugar con la "base" o "fundación". Este dominio consiste en los intereses compartidos por una comunidad, e incluye los medios de producción (como la tierra y el agua), bienes y constructos ideológicos, como el conocimiento, la tecnología, leyes, prácticas, habilidades y costumbres... Un segundo dominio está constituido por las relaciones sociales y las asociaciones. Estas relaciones comunales son vistas como un fin en sí mismas e incluyen economías domésticas, corporaciones de diversos tipos, linajes y naciones...El tercer dominio está constituido por los bienes y servicios intercambiados en el mercado. En este dominio los bienes y los servicios están separados de las relaciones sociales, se busca el máximo beneficio y se ayuda a la circulación mediante monedas o monedas de uso parcial comunalmente fabricadas...El cuarto dominio, por último, lo constituye la apropiación y acumulación de riqueza, llevada a cabo por múltiples instituciones, no necesariamente especializadas únicamente en este dominio: jefes de linaje, estados, corporaciones, bancos, etc." (Molina \&
Valenzuela, 2004, pág. 70)

Estos cuatro dominios se relacionan con la propuesta de súper estructura e infraestructura del marxismo y puede contrastarse con las distintas implicaciones del fenómeno económico que se desarrollan en el contexto, la vinculación de las familias con los aspectos naturales, especialmente con la tierra y el agua, las organizaciones, políticas religiosas y lo generación de ingresos por medio de la oferta y demanda de bienes y servicios dentro de la comunidad, y hacia los núcleos urbanos, estos dominios son coincidentes con las necesidades de la comunidad más allá del espacio físico- políticos y que incluye aquellos aspectos de carácter cultural que nos aporta a entender la verdadera dimensiones de la comunidad y por lo tanto de la economía comunitaria.

Otro aspecto importante para entender las verdaderas dimensiones, potencialidades y limitante de la economía comunitaria es lo relacionado con su vinculación con otros ámbitos de la economía, especialmente el papel del gobierno y las políticas hacia este sector. Esto es determinante en tanto posiciona a la comunidad campesina como fuente de riqueza y sector importante o bien como imagen del atraso que se olvida o desaparece, de estas dos modelos y sus intermedios está plagada la historia latinoamericana, desde las reformas agrarias y la revoluciones obreras hasta la promoción de los latifundios y la persecución del campesinado, en este sentido:

"La integración de la economía familiar campesina a las actividades de crédito, procesamiento de alimentos, acopio y comercialización, gestión de recursos, incidencia en las políticas económicas y sociales del gobierno, es parte importante de la estrategia para que el campesinado pueda acceder a los excedentes de la riqueza nacional, siendo como es su principal generador" (Font, 2010)

Gran parte de las problemáticas económicas de las familias campesinas están asociadas directamente con su relación desigual con la economía local, nacional e internacional, por ejemplo la banca privada no considera el cultivo de patio o la pequeña producción agrícola como sujeta de crédito y la pequeña producción agrícola se ve limitada por aspectos como el trasporte, trabas legales o incapacidad de acopio de las familias.

En el actual contexto una referencia importante es el programa Bono Productivo Alimentario "Hambre 
Cero" que ha desarrollado el Gobierno de Nicaragua desde el año 2007 y consiste en la entrega de animales de crianza, insumos materiales y capacitación para la crianza y producción. Este programa ha sido destacado por la FAO que en varios informes destacó el avance en la reducción de la desnutrición y el alcance de las metas propuestas en los objetivos del milenio (FAO, 2014) mientras que muchos críticos del Gobierno y del programa aducían que una vaca o una gallina no podría marcar la diferencia en cuanto al desarrollo del sector rural. El gobierno entregó miles de bonos y beneficios que capitalizaron las familias campesinas y generaron un efecto positivo en las comunidades, este hecho ampliamente documentado demuestra la eficacia de las políticas de capitalización de la familia campesina.

\section{Conclusiones}

$\mathrm{Al}$ conocer las dinámicas culturales propias de la comunidad campesina asociadas al cultivo de patio, es posible comprender y valorizar en sus amplias dimensiones algunos elementos de la economía comunitaria y su relación con los procesos identitarios y productivos como el uso de la tierra, las plantas, árboles y sus usos cotidianos, asociados a prácticas festivas, alimenticias y de intercambio que mejoran sustancialmente la calidad de vida de las poblaciones.

Por otro lado la crianza de animales, el cuido de plantas y los roles que se desarrollan en torno a estas tareas, entre otras, juegan un papel importante en la preservación de la identidad campesina que se ha trastocado debido a los procesos de migración, cambios en las actividades laborales y económicas, entre otras. Experticias locales y nacionales demuestran la urgencia e importancia de proponer programas o proyectos que capitalicen los saberes de la identidad campesina para la producción económica y la reproducción social.

Es importante comprender el papel de las relaciones sociales en la economía comunitaria y sus vínculos con elementos externos a ella, específicamente el papel del Gobierno y el mercado local y regional para el desarrollo comunitario, pero también para el bienestar de la población del resto del país y el desarrollo nacional. En este sentido se ha podido observar la forma importante en que la economía comunitaria aporta a la reducción de la pobreza y la seguridad alimentaria.

\section{Bibliografía}

FAO. (2014). Panorama de la seguridad alimentaria y nutricional en Centroamerica y Republica Dominicana. Panama: FAO.

Font, E. (16 de 12 de 2010). http://www.rebelion. org. Recuperado el 19 de 03 de 2014, de Rebelion: http://www.rebelion.org/noticia. php?id=118766

Guber, R. (2005). El salvaje metropolitano . Buenos Aires: PAIDOS.

INIDE. (2014). Encuesta de Medición de Nivel de Vida 2014. Managua: Gobierno de Nicaragua.

INIFOM. (24 de junio de 2014). inifom. Obtenido de http://www.inifom.gob.ni/municipios/documentos/MASAYA/masatepe.pdf.

Jimeno, M. (2000). La emergencia del investigador ciudadano: estilos de antropología y crisis de modelos en la antropología colombiana. En J. Tocancipa, La formación del estado nación y las disciplinas sociales en Colombia (pág. 163). Popayan: taller editorial.

López Mercado, S. (05 de mayo de 2014). Historia de la cooperativa. (X. R. Corea, Entrevistador)

Martín, J. R. (2009). Observación Participante: ¿técnica o método? Nure Investigación, $n^{\circ} 39,5$.

Molina, J. L., \& Valenzuela, H. (2004). Invitación a la antropología económica. Barcelona: UAB.

Narotzky, S. (2004). Antropología Económica. Madrid: melusina.

Palerm, J. (1996). Los nuevos campesinos. Mexico: paidos.

Perez Gutierrez, N. (28 de abril de 2014). Sobre la producción agrícola y la organización de la familia. (X. Rodriguez, Entrevistador) 
Pinto, J. S. (2007). Antropología económica en el contexto rural contemporáneo. Revista de Antropología Experimental no 7, 63- 69.

Sevilla Aleman, V. (30 de abril de 2014). Liderazgo en GRUDESA. (X. Rodriguez, Entrevistador)

Taylor, s., \& Bogdan, R. (1998). Introducción a los metodos cualitativos de investigación. Barcelona: PAIDOS. 